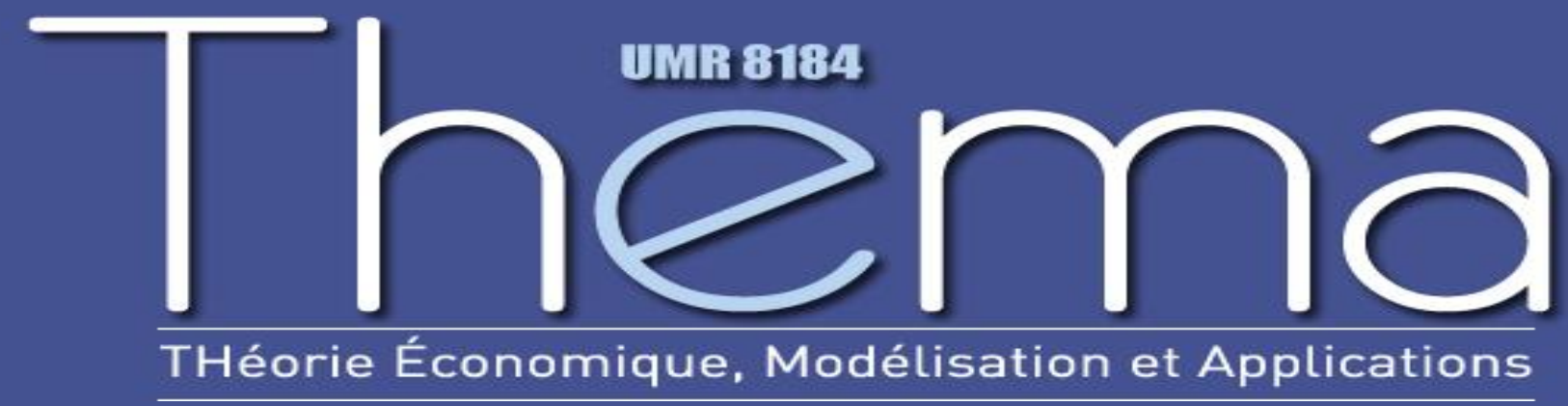

Thema Working Paper $\mathrm{n}^{\circ}$ 2011-17

Université de Cergy Pontoise, France

On the likelihood of Dummy players in Weighted Majority Games

Fabrice Barthelemy Dominique lepelley Mathieu Martin

June, 2011 


\title{
On the Likelihood of Dummy Players in Weighted Majority Games
}

Fabrice BARTHÉLÉMY*, Dominique LEPELLEY ${ }^{\dagger}$ and Mathieu MARTIN $\ddagger$

\begin{abstract}
When the number of players is small in a weighted majority voting game, it can occur that one of the players has no influence on the result of the vote, in spite of a strictly positive weight. Such a player is called a "dummy" player in game theory. The purpose of this paper is to investigate the conditions that give rise to such a phenomenon and to compute its likelihood. It is shown that the probability of having a dummy player is surprisingly high and some paradoxical results are observed.
\end{abstract}

JEL classification: C7, D7

Keywords: Cooperative game theory, weighted voting games, dummy player, likelihood of voting paradoxes.

*THEMA, Université de Cergy Pontoise, 33 boulevard du Port, 95011 Cergy Pontoise Cedex, FRANCE. E-mail: fabrice.barthelemy@u-cergy.fr

†CEMOI, Université de la Réunion, 97715 Saint Denis cedex 9, FRANCE. E-mail: dominique.lepelley@univ-reunion.fr

†THEMA, Université de Cergy Pontoise, 33 boulevard du Port, 95011 Cergy Pontoise Cedex, FRANCE. E-mail: mathieu.martin@u-cergy.fr 


\section{Introduction}

The main teaching of the literature on power indices is that, in a collective choice process, voting power or influence need not to be proportional to the relative number of votes (weight) an individual or a group (player) is entitled to. An extreme and striking consequence of this non proportionality is that a player can have a positive weight but never be a member of a minimal winning coalition (a coalition that wins and the removal of a single player does not allow the coalition to win any longer). Such players have no voting power and are known as dummies.

The most famous example of this somewhat paradoxical phenomenon is offered by Luxembourg in the Council of Ministers of the EU between 1958 and 1973. Luxembourg held one vote, whereas the quota for a proposition to be approved was 12 out of 17 . Since other member states held an even number of votes (4 for Germany, France and Italy, 2 for Belgium and The Netherlands), Luxembourg formally was never able to make any difference in the voting process and was a dummy.

Another well known case of dummies involves Nassau County, New York. Nassau County's government took the form of a Board of Supervisors, one representative for each of various municipalities, who cast a block of votes. Here are the weighted voting systems used at various times by Nassau County. The passing quota shown reflects the number of votes needed to pass "ordinary legislation".

\begin{tabular}{|c|c|c|}
\hline & 1958 & 1964 \\
\hline Hempstead 1 & 9 & 31 \\
Hempstead 2 & 9 & 31 \\
North Hempstead & 7 & 28 \\
Oyster Bay & 3 & 21 \\
Long Beach & 1 & 2 \\
Glen Cove & 1 & 2 \\
\hline Total votes & 30 & 115 \\
\hline Quota & 16 & 58 \\
\hline
\end{tabular}

The numerical weights were chosen to try to take into account the populations of the different municipalities, which were quite disparate. It is easy to see that in 1958, Oyster Bay, Long Beach and Glen Cove were dummies. It can also be checked that, in 1964, there were two dummies (Glen Cove and Long Beach). After 1964, the quota was raised to guarantee that no municipality was a dummy.

A third example of dummy has recently been discovered by one of the authors (see Blancard and Lepelley, 2010) in a community of municipalities in La R?union (France). This community, called CIVIS (Communaut? Intercommunale des VIlles Solidaires), gathers five municipalities: Saint-Pierre (15 representatives in the community council), Saint-Louis (10 representatives), L'Etang-Sal? (4), Petite-?le (4) and Cilaos (3), the number of representatives being roughly proportional to the municipality population. In the community Council, 19 votes are necessary 
for a proposition to be accepted. If we suppose that the representatives of a municipality vote as a block, it can be seen that Cilaos is a dummy: all the winning coalitions containing Cilaos remain winning when this municipality is removed.

The possibility of dummy players is clearly problematic from a democratic point of view and the diversity of the examples given above suggests that the occurrence of dummies in voting games is of practical concern and could be less rare that expected in first analysis. What is the likelihood of such an undesired phenomenon? How the distribution of weights should be arranged in order to avoid the occurrence of dummies in voting games?

We propose in this paper a theoretical investigation of these issues in the context of weighted majority games, where the quota is equal to the half of the total number of votes, plus one. Our framework and our main assumptions are introduced in Section 2. We propose some analytical results in Section 3 for weighted voting games with 4, 5 and 6 players: in each case, we characterize the distributions of weights giving rise to the occurrence of the "dummy paradox" and deduce from these characterizations some representations for the likelihood of the paradox as a function of the total number of votes. Section 4 proposes exact numerical results for the likelihood of dummy players for more than 6 players and for some specified values of the total number of votes. Our results are discussed in Section 5, where we study the impact of a reduction of the weight scattering on the probability of having some dummies. Section 6 concludes the paper.

\section{Framework and assumptions}

We will adopt the following notation:

$m$ is the number of players (or voters). The players are denoted by $J 1, J 2, \ldots, J m . N$ is the set of all players and a subset of $N$ is called a coalition.

$n_{i}$ is the weight of player $i$ and $n=\sum_{i} n_{i}$. Hence, $n_{i}$ can be interpreted as the number of votes assigned to a member $J i$ of a voting body. Notice however that, when the players are parties in a political assembly, the $n_{i}$ 's correspond to the number of representatives of each party and $n$ is the total number of votes in the assembly.

As mentioned above, we only consider in the present study Weighted Majority Games (WMG): a proposition is adopted if and only if the total weight of the players in favor of this proposition is greater or equal to $n / 2+1$ if $n$ is even and to $(n+1) / 2$ if $n$ is odd. In what follows, this majority quota will be denoted by $Q=[n / 2]^{+}$, where $[x]^{+}$is the smallest integer strictly higher than $x$. So, a coalition $S$ is winning if and only if $\sum_{i \in S} n_{i} \geq Q$; otherwise, the coalition is said to be loosing. ${ }^{1}$ A player $J i$ is a dummy if and only if, for each winning coalition $S$ including $J i, S-\{J i\}$ is still winning.

Our main assumptions are the following:

\footnotetext{
${ }^{1}$ Notice that, when $n$ is even, the complementary coalition of a loosing coalition is not always winning. In order to take into account this peculiarity, we will make use of the following notation: $Q^{*}=Q$ if $n$ is odd and $Q^{*}=Q-1$ if $n$ is even.
} 
(1) the $n_{i}$ 's are integer,

(2) $n / 2 \geq n_{1} \geq n_{2} \geq \ldots \geq n_{m} \geq 1$,

(3) $m$ and $n$ being given, all the distributions of the $n_{i}$ 's verifying (1), (2) and $n=\sum_{i} n_{i}$ are equally likely to occur.

Notice that this framework fits well with the (recent) French local entities called EPCI (Etablissement Public de Cooperation Intercommunale) where each municipality belonging to the EPCI is given a number of delegates approximately proportionate to its number of inhabitants $^{2}$. In this context, $n_{1}$ is the number of delegates of the biggest municipality in the EPCI council, $n_{m}$ the number of delegates of the smallest, and $n$ is the total number of delegates in the EPCI council (we suppose that, in this council, the delegates of a given municipality vote as a bloc). Of course, the biggest municipality should not be a dictator $\left(n_{1} \leq n / 2\right)$ and the smallest one should obtain at least one delegate. In the EPCI council, the current decisions are taken with a quota $Q=[n / 2]^{+}$.

\section{Some analytical results}

Proposition 1 In a m-player WMG, (i) the maximum number of possible dummies is equal to $m-3$ and (ii) the number of dummies is exactly $m-3$ if and only if $n_{2}+n_{3} \geq Q$.

Proof. In order to prove $(i)$, we have to show that $J 3$ cannot be a dummy in a $m$-player majority game, $m \geq 3$. Suppose the contrary: J3 is a dummy. A first consequence is that $J 4, J 5, \ldots, J m$ are also dummies. Furthermore, the coalition $\{J 1, J 3\}$ is loosing (if this coalition was winning, the fact that $J 3$ is a dummy would imply that $n_{1}>n / 2$, contradicting our assumptions). Now, if $\{J 1, J 3\}$ is loosing, then $\{J 1, J 3, J 4\}$ is also loosing since $J 4$ is a dummy. Similarly, as $J 5$ is a dummy, the coalition $\{J 1, J 3, J 4, J 5\}$ is loosing and we can set in the same way that $\{J 1, J 3, J 4, \ldots, J m\}$ is loosing, which implies $n_{1}+n_{3}+n_{4}+n_{5}+\ldots+n_{m}<Q$. As $\sum_{i} n_{i}=n$, we would have $n_{2} \geq n / 2$, which is impossible.

Consider now assertion $(i i)$ and suppose that $n_{2}+n_{3} \geq Q$. Let's show this implies that $J 4$ is a dummy. Consider the winning coalitions including $J 4$. Observe first that $n_{2}+n_{3} \geq Q$ implies that the coalition $\{J 4, J 5, \ldots, J m\}$ is loosing. Observe next that the coalition $\{J 1, J 4\}$ is also loosing: $n_{2}+n_{3} \geq Q$ implies $n_{1}+n_{4}+n_{5}+\ldots+n_{m}<Q$, which implies $n_{1}+n_{4}<Q$. It follows from these observations that the only winning coalitions with $J 4$ must include two players among $\{J 1, J 2, J 3\}$. As $n_{2}+n_{3} \geq Q$ and $n_{1} \geq n_{2} \geq n_{3}$, we have $n_{1}+n_{3} \geq Q$ and $n_{1}+n_{2} \geq Q$. Consequently, the defection of $J 4$ in these coalitions lets them winning and $J 4$ is a dummy.

Finally, suppose that $J 4$ is a dummy. This implies that the coalition $\{J 1, J 4\}$ is loosing (if not, $J 4$ dummy would imply $n_{1} \geq Q$, a contradiction). As $J 5, J 6, \ldots, J m$ are (also) dummies, it follows that $\{J 1, J 4, J 5\},\{J 1, J 4, J 5, J 6\}, \ldots,\{J 1, J 4, J 5, J 6, \ldots, J m\}$ are also loosing. But $\{J 1, J 4, J 5, J 6, \ldots, J m\}$ loosing implies $n_{1}+n_{4}+n_{5}+\ldots+n_{m}<Q$ and, consequently, $n_{2}+n_{3} \geq$

\footnotetext{
${ }^{2}$ The CIVIS we have mentioned in the Introduction is an example of EPCI.
} 
$Q^{*}$. As $J 4$ dummy makes $n_{2}+n_{3}=Q^{*}$ impossible in the case where $n$ is even, we finally conclude that we must have $n_{2}+n_{3} \geq Q$.

Corollary 1 There is no dummy player in a 3-player WMG and, in a 4-player WMG, J4 is a dummy player if and only if $n_{2}+n_{3} \geq Q$.

The following proposition deals with 5-player and 6-player WMG's.

Proposition 2 (i) In a 5-player WMG, J5 is a dummy player if and only if one of the following cases holds:

- case 1: $n_{2}+n_{3}+n_{4} \geq Q$ and $n_{1}+n_{4} \geq Q$;

- case 2: $n_{2}+n_{3} \geq Q$.

In case 2 (and only in this case), both J4 and J5 are dummy players.

(ii) In a 6-player WMG, J6 is a dummy player if and only if one of the following cases holds:

- case 1: $n_{2}+n_{3}+n_{4}+n_{5} \geq Q$ and $n_{1}+n_{5} \geq Q$;

- case 2: $n_{2}+n_{3}+n_{4} \geq Q$ and $n_{1}+n_{4} \geq Q$;

- case 3: $n_{2}+n_{3}+n_{5} \geq Q$ and $n_{1}+n_{4}+n_{5} \geq Q$ and $n_{1}+n_{3} \geq Q$;

- case $4: n_{2}+n_{3} \geq Q$

- case 5: $n_{2}+n_{4}+n_{5} \geq Q$ and $n_{1}+n_{2} \geq Q$;

- case 6: $n_{3}+n_{4}+n_{5} \geq Q$.

In case 2, J5 and J6 are dummy players; in case 4, J4, J5 and J6 are dummy players.

The proof of this proposition is rather tedious and is given in Appendix.

Corollary 1 and Proposition 2 allow us to enumerate the distributions of the weights that give rise to dummy players and to compute the probability of their occurrence in $m$-player WMG's, with $m \in\{4,5,6\}$. Moreover, it is possible to derive from Corollary 1 and Proposition 2 some representations for this probability as a function of $n$, the total number of votes. This probability is denoted by $P(m, n)$ in what follows.

Proposition 3 For $n \equiv 9$ modulo 12, the probability of having a dummy player in a 4-player $W M G$ is given as:

$$
P(4, n)=\frac{n^{2}-33}{2\left(n^{2}+3 n-12\right)} .
$$

As a consequence, $\lim _{n \rightarrow \infty} P(4, n)=\frac{1}{2}$.

Proof. Given our assumption (3) and for a given value of $n$, we have to divide the number of those distributions of the $n_{i}$ 's that give rise to the occurrence of a dummy player (denoted by $D(4, n))$ by the total number of possible distributions with 4 players (denoted by $T(4, n)$ ). We begin by evaluating $T(4, n)$. A vector of integers $\left(n_{1}, n_{2}, n_{3}, n_{4}\right)$ is a possible distribution of the weights is and only if

$$
n_{1} \geq n_{2}, n_{2} \geq n_{3}, n_{3} \geq n_{4}, n_{4} \geq 1, n_{1} \leq n / 2 \text { and } n_{1}+n_{2}+n_{3}+n_{4}=n \text {. }
$$

We know from Ehrhart's theory and its recent developments (the reader is referred to Lepelley et al. (2008) for a presentation of this theory) that the number of integer solutions of such a set 
of (in)equalities is a quasi polynomial in $n$ with periodic coefficients (or Ehrhart's polynomial). A periodic coefficient takes various values according to $n$ and to a given period. For example, $c(n)=\left[\frac{1}{2}, \frac{3}{4}, 1\right]_{n}$ is a periodic coefficient with period $3, c(n)=\frac{1}{2}$ if $n \equiv 0$ modulo $3, c(n)=\frac{3}{4}$ if $n \equiv 1$ modulo 3 and $c(n)=1$ if $n \equiv 2 \operatorname{modulo} 3$. Numerous algorithms exist to derive the expression of such a quasi polynomial (see, once again, Lepelley et al. (2008)). Using one of these algorithms, we obtain

$T(4, n)=\frac{1}{288} n^{3}+\left[\frac{1}{32}, \frac{1}{48}\right]_{n} n^{2}+\left[\frac{1}{24},-\frac{1}{96}\right]_{n} n+\left[0,-\frac{1}{72},-\frac{17}{72},-\frac{1}{4}, \frac{1}{9}, \frac{7}{72},-\frac{1}{8},-\frac{5}{36},-\frac{1}{9},-\frac{1}{8},-\frac{1}{72},-\frac{1}{36}\right]_{n}$. The period of such a quasi polynomial is the least common multiple of the periods of its coefficients, here 12. Consequently, the expression of $T(4, n)$ corresponds to 12 distinct polynomials; for instance, we obtain for $n \equiv 9 \operatorname{modulo} 12:^{3}$

$$
T(4, n)=\frac{1}{288} n^{3}+\frac{1}{48} n^{2}-\frac{1}{96} n-\frac{1}{8}=\frac{(n+3)\left(n^{2}-33\right)}{576} .
$$

Now, according to Corollary 1, a dummy player exists if and only if

$$
n_{1} \geq n_{2}, n_{2} \geq n_{3}, n_{3} \geq n_{4}, n_{4} \geq 1, n_{1} \leq n / 2, n_{2}+n_{3}>n / 2 \text { and } n_{1}+n_{2}+n_{3}+n_{4}=n .
$$

The number of associated distributions of the $n_{i}$ 's is given as

$D(4, n)=\frac{1}{576} n^{3}+\left[-\frac{1}{96}, \frac{1}{192}\right]_{n} n^{2}+\left[-\frac{1}{24},-\frac{11}{192}, \frac{1}{48}, \frac{1}{192}\right]_{n} n+\left[0, \frac{29}{576},-\frac{7}{72},-\frac{7}{64}, \frac{2}{9},-\frac{35}{576},-\frac{1}{8}, \frac{65}{576}, \frac{1}{9},-\frac{11}{64}\right.$, $\left.\frac{7}{72}, \frac{1}{576}\right]_{n}$,

which implies, for $n \equiv 9$ modulo 12 :

$$
D(4, n)=\frac{1}{576} n^{3}+\frac{1}{192} n^{2}-\frac{11}{192} n-\frac{11}{64}=\frac{(n+3)\left(n^{2}+3 n-12\right)}{288} .
$$

The expression of $P(4, n)=D(4, n) / T(4, n)$ for $n \equiv 9$ modulo 12 directly follows, as well as the limiting value ${ }^{4} P(4, \infty)=\frac{\frac{1}{576}}{\frac{1}{288}}=\frac{1}{2}$.

The two following Propositions are obtained along the same lines as Proposition 3 and their proofs are omitted.

Proposition 4 For $n \equiv 15$ modulo 120, the probability of having dummy player(s) in a 5player $W M G$ is:

$$
P(5, n)=\frac{5(n+9)\left(7 n^{3}-51 n^{2}+165 n-801\right)}{6\left(11 n^{4}+120 n^{3}+350 n^{2}+960 n+4815\right)} .
$$

Consequently, the probability for P5 to be a dummy when $n$ is large is: $\lim _{n \rightarrow \infty} P(5, n)=\frac{35}{66}$. And the limiting probability of having two dummy players (J4 and J5) when $n$ is large is given as $\frac{5}{22}$.

Proposition 5 The limiting probability of having at least one dummy player in a 6-player $W M G$ is given by: $\lim _{n \rightarrow \infty} P(6, n)=\frac{155}{312}$. The limiting probability of having two dummies (J5 and J6) is $\frac{5}{39}$ and the limiting probability of having three (J4, J5 and J6) is $\frac{5}{52}$.

\footnotetext{
${ }^{3}$ Of course, the 11 other polynomials can be derived in the same way.

${ }^{4}$ It is worth noticing that the coefficient of the leading term of the quasi polynomials is not periodic. This peculiarity allows to easily obtain the desired probabilities for $n$ large by considering only this coefficient in the quasi plolynomials.
} 
Table 1

Probability $P(m, n)$ of having a dummy player

as a function of $n$ (the total number of votes) for $m=4,5,6$.

\begin{tabular}{|c|c|c|c|}
\hline$n$ & 4-player WMG & 5-player WMG & 6-player WMG \\
\hline 15 & 0.4375 & 0.2609 & 0.1818 \\
\hline 18 & 0.2258 & 0.1778 & 0.0196 \\
\hline 21 & 0.4146 & 0.2973 & 0.1978 \\
\hline 24 & 0.2537 & 0.2302 & 0.0529 \\
\hline 27 & 0.4578 & 0.3696 & 0.2731 \\
\hline 30 & 0.3089 & 0.2827 & 0.1002 \\
\hline 33 & 0.4490 & 0.3818 & 0.2905 \\
\hline 36 & 0.3235 & 0.3087 & 0.1259 \\
\hline 39 & 0.4684 & 0.4145 & 0.3310 \\
\hline 42 & 0.3535 & 0.3398 & 0.1601 \\
\hline 45 & 0.4637 & 0.4213 & 0.3407 \\
\hline 48 & 0.3624 & 0.3553 & 0.1809 \\
\hline 51 & 0.4747 & 0.4402 & 0.3641 \\
\hline 54 & 0.3813 & 0.3757 & 0.2072 \\
\hline 57 & 0.4718 & 0.4443 & 0.3711 \\
\hline 60 & 0.3873 & 0.3859 & 0.2232 \\
\hline 63 & 0.4789 & 0.4564 & 0.3869 \\
\hline 66 & 0.4002 & 0.4002 & 0.2431 \\
\hline 69 & 0.4770 & 0.4593 & 0.3918 \\
\hline 72 & 0.4045 & 0.4075 & 0.2558 \\
\hline 75 & 0.4820 & 0.4678 & 0.4028 \\
\hline 78 & 0.4139 & 0.4181 & 0.2716 \\
\hline 81 & 0.4806 & 0.4699 & 0.4066 \\
\hline 84 & 0.4172 & 0.4235 & 0.2818 \\
\hline 87 & 0.4842 & 0.4761 & 0.4149 \\
\hline 90 & 0.4243 & 0.4316 & 0.2943 \\
\hline 93 & 0.4832 & 0.4777 & 0.4177 \\
\hline 96 & 0.4269 & 0.4359 & 0.3027 \\
\hline 99 & 0.4860 & 0.4825 & 0.4241 \\
\hline 199 & 0.4928 & 0.5061 & 0.4594 \\
\hline 202 & 0.4645 & 0.4624 & 0.3935 \\
\hline limit & $1 / 2$ & $\frac{35}{66}=0.530$ & $\frac{155}{312}=0.497$ \\
\hline
\end{tabular}




\section{Table 2}

\section{Probability $P(m, \infty)$ of having one, two or three dummies}

for $m=4,5,6$.

\begin{tabular}{||c||c|c|c|c||}
\hline$m$ & 1 dummy & 2 dummies & 3 dummies & Total \\
\hline 4 & 0.5 & 0 & 0 & 0.5 \\
5 & 0.3030 & 0.2273 & 0 & 0.5303 \\
6 & 0.2724 & 0.1282 & 0.0962 & 0.4968 \\
\hline
\end{tabular}

The next section deals with the cases with more than six players.

\section{Results for more than six players}

In the 5-player case, 120 different formulas are necessary to compute all the probabilities $P(5, n)$ (see Proposition 4 for one of them). The number of different formulas is exponentially increasing when $m$ increases and it becomes practically too complicated to list all of them when $m$ is higher than 6 . In order to obtain the desired probabilities for more than 6 players, we make use of a computer. This is done in two ways: exact computations and simulations. Exact computations are done with an exhaustive list of all the possible vectors of weights for a given number $n$ of votes in the assembly. For all these vectors $\left(n_{1}, \ldots, n_{m}\right)$, we check whether or not the last player is pivotal (decisive) (remember that $n_{1} \geq n_{2} \geq \ldots \geq n_{m}$ ). This is done by computing the Banzhaf powr index with the generating function method. Finally, the exact probability of having at least one dummy player is the ratio between the number of times the last player $J_{m}$ is never pivotal (decisive) and the number of vectors $\left(n_{1}, \ldots, n_{m}\right)$ considered as admissible (with a uniform distribution of weights vectors, as done theoretically in the previous section).

\section{Table 3}

Probability $P(m, n)$ of having a dummy player

as a function of $m$ for $n=45, n=50, n=95$ and $n=100$.

\begin{tabular}{|c||c|c|c|c||}
\hline$m$ & $n=45$ & $n=50$ & $n=95$ & $n=100$ \\
\hline 4 & 0.4637 & 0.3735 & 0.4855 & 0.4297 \\
5 & 0.4213 & 0.3020 & 0.4806 & 0.4003 \\
6 & 0.3407 & 0.1931 & 0.4215 & 0.3091 \\
7 & 0.2135 & 0.0858 & 0.3173 & 0.1869 \\
8 & 0.1050 & 0.0299 & 0.2017 & 0.0862 \\
9 & 0.0434 & 0.0091 & 0.0963 & 0.0304 \\
10 & 0.0185 & 0.0030 & 0.0447 & 0.0108 \\
11 & 0.0086 & 0.0012 & $0.0194^{*}$ & $0.0044^{*}$ \\
12 & 0.0044 & 0.0005 & $0.0098^{*}$ & $0.0017^{*}$ \\
13 & 0.0021 & 0.0002 & $0.0060^{*}$ & $0.0008^{*}$ \\
14 & 0.0007 & 0.0000 & $0.0038^{*}$ & $0.0005^{*}$ \\
15 & 0.0004 & 0.0000 & $0.0025^{*}$ & $0.0003^{*}$ \\
\hline
\end{tabular}

*Simulated probabilities 
Our simulations are based on random vectors of weights. The estimated probability of having at least one dummy player is then obtained by dividing the number of times the last player $J_{m}$ is never pivotal (decisive) by the number of vectors $\left(n_{1}, \ldots, n_{m}\right)$ randomly generated.

Table 4

Simulated $^{5}$ probability $P(\infty, n)$ of having one, two ... or $x$ dummies

\begin{tabular}{||c||c||c|c|c|c|c||}
\hline \multicolumn{1}{||c||}{} & \multicolumn{7}{c||}{ Number of dummy players } \\
\hline$m$ & $\geq 1$ & 1 & 2 & 3 & 4 & 5 \\
\hline 4 & 0.49300 & 0.49300 & & & & \\
5 & 0.52490 & 0.29830 & 0.22660 & & & \\
6 & 0.49310 & 0.27140 & 0.12750 & 0.09240 & & \\
7 & 0.43530 & 0.24530 & 0.10460 & 0.04570 & 0.03980 & \\
8 & 0.34470 & 0.21030 & 0.07970 & 0.02930 & 0.01300 & 0.01240 \\
9 & 0.25750 & 0.17250 & 0.04930 & 0.01960 & 0.00790 & 0.00370 \\
10 & 0.17750 & 0.13160 & 0.02710 & 0.01000 & 0.00340 & 0.00230 \\
11 & 0.11844 & 0.09634 & 0.01440 & 0.00414 & 0.00138 & 0.00074 \\
12 & 0.07140 & 0.06044 & 0.00756 & 0.00184 & 0.00058 & 0.00024 \\
13 & 0.04340 & 0.03940 & 0.00294 & 0.00062 & 0.00020 & 0.00004 \\
14 & 0.02282 & 0.02132 & 0.00114 & 0.00024 & 0.00004 & $0^{+}$ \\
15 & 0.01226 & 0.01168 & 0.00046 & 0.00004 & 0.00002 & $0^{+}$ \\
\hline
\end{tabular}

$0^{+}$: the estimated probability is less than $1 / 50000$

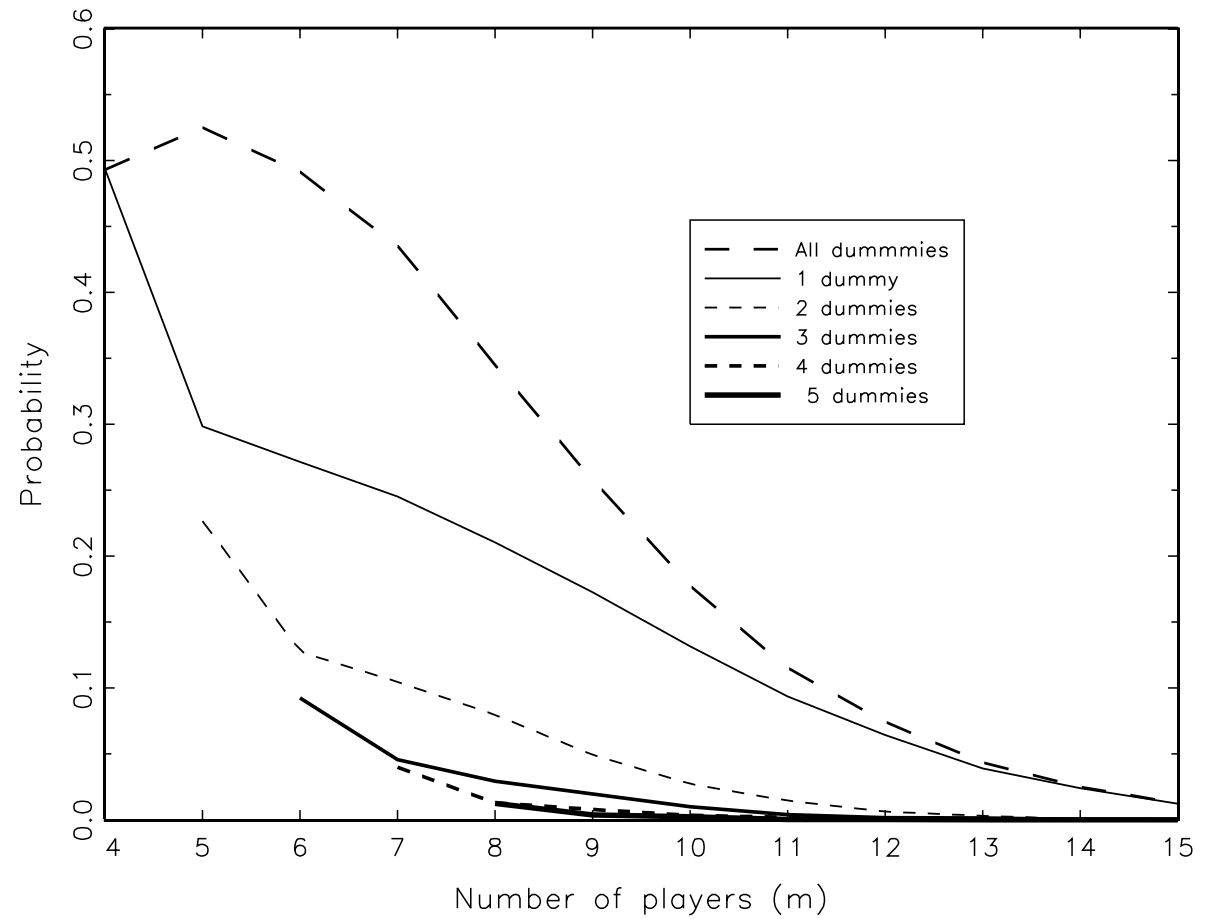

Figure 1. Simulated Probability $P(\infty, n)$ of having exactly $x$ dummies with $m$ players

\footnotetext{
${ }^{5} 10000$ simulations for $m<11$ and 50000 when $m \geq 11$. Moreover $P(\infty, n)$ estimating $P(99999, n)$.
} 


\section{Discussion and further results}

The theoretical risk of having a dummy appears to be very high. It can be suggested that our calculations possibly overestimate this risk in the case of the french EPCI, which very often try to reduce the spread (range) of the numbers of representatives in each city. How can we introduce more realism in our analysis?

One approach is the following. Let $k$ be the maximal fraction of the total weight given to the "biggest" player: $n_{1} / n \leq k$. We wish to study the impact of parameter $k$ on the probability of having a dummy player. Under our other assumptions, $k$ belongs to $\left[\frac{1}{m}, \frac{1}{2}\right]$. When $k=\frac{1}{2}$, we recover the situation we have studied in the preceding sections. With $k=\frac{1}{m}$, each player obtains the same weight, hence the same power and there is no dummy. Let $P(m, n, k)$ be the probability of having a dummy when $J 1$ gets $k \%$ of the total weight. It seems natural to conjecture that $P(m, n, k)$ decreases when $k$ moves from $\frac{1}{2}$ to $\frac{1}{m}$. The following results show that this conjecture does not hold for small values of $m$. We will only give the proof of the first Proposition. ${ }^{6}$

Proposition 6 In a 4-player WMG with $n$ large, the probability of having a dummy player as a function of $k$ is given by the following representation:

$$
\begin{gathered}
P(4, \infty, k)=\frac{3}{4} \text { for } \frac{1}{4} \leq k<\frac{1}{3} \\
=\frac{240 k^{3}-288 k^{2}+108 k-13}{4\left(44 k^{3}-60 k^{2}+24 k-3\right)} \text { for } \frac{1}{3} \leq k \leq \frac{1}{2} .
\end{gathered}
$$

Proof. Let $K$ be the maximal weight of $J 1$, with $k=K / n$. In order to compute the desired probability, we begin by evaluate the total number $T(4, n, K)$ of distributions on the $n_{i}$ 's when $n_{1}$ is constrained to be lower or equal to $K . T(4, n, K)$ is the number of integer solutions of the following inequalities :

$$
n_{1} \geq n_{2}, n_{2} \geq n_{3}, n_{3} \geq n_{4}, n_{4} \geq 1, n_{1} \leq K \quad n_{1}+n_{2}+n_{3}+n_{4}=n \text { and } K \leq n / 2 .
$$

We have now two parameters, $n$ and $K$, and the number of integer solutions is given by bivariate quasi polynomials (see Lepelley et al.(2008)). Using an algorithm recently developed by Barvinok (????), we obtain for $n$ even two distinct quasi polynomials associated with two validity domains:

For $\frac{n}{4} \leq K<\frac{n}{3}$ :

$T(4, n, K)=-\frac{1}{144} n^{3}+\left(\frac{1}{12} K+\frac{5}{48}\right) n^{2}+\left(-\frac{1}{3} K^{2}-\frac{5}{6} K-\frac{1}{2}\right) n+\frac{4}{9} K^{3}+\frac{5}{3} K^{2}+2 K+c_{1} ;$

For $\frac{n}{3} \leq K \leq \frac{n}{2}$ :

$T(4, n, K)=\frac{1}{48} n^{3}+\left(-\frac{1}{6} K-\frac{3}{16}\right) n^{2}+\left(\frac{5}{12} K^{2}+-\frac{11}{12} K+\frac{5}{12}\right) n-\frac{11}{36} K^{3}-\frac{23}{24} K^{2}-2 K+c_{2}$, where $c_{1}$ and $c_{2}$ are periodic constants the value of which depends on both $n$ and $K$. Consider the first domain. As $K=k n$, it follows that, for $\frac{n}{4} \leq K<\frac{n}{3}$, i.e. for $\frac{1}{4} \leq k<\frac{1}{3}$ : $T(4, n, k)=-\frac{1}{144} n^{3}+\left(\frac{1}{12} k n+\frac{5}{48}\right) n^{2}+\left(-\frac{1}{3} k^{2} n^{2}-\frac{5}{6} k n-\frac{1}{2}\right) n+\frac{4}{9} k^{3} n^{3}+\frac{5}{3} k^{2} n^{2}+2 k n+c_{1}$

${ }^{6}$ Although more cumbersome, the proofs of Proposition 7 and 8 are quite similar. 
$=\left(-\frac{1}{144}+\frac{1}{12} k-\frac{1}{3} k^{2}+\frac{4}{9} k^{3}\right) n^{3}+\left(\frac{5}{48}-\frac{5}{6} k+\frac{5}{3} k^{2}\right) n+\left(-\frac{1}{2}+2 k\right) n+c_{1}$.

Observe that, in order to compute the limiting probability $P(4, \infty, k)$, only the coefficient of the leading term in $n^{3}$ matters. For this reason, we will only give the coefficient of $n_{3}$ of the quasi polynomials we exhibit in the remaining of this proof.

Proceeding as above, we obtain for the second domain, $\frac{1}{3} \leq k \leq \frac{1}{2}$ :

$T(4, n, k)=\left(\frac{1}{48}-\frac{1}{6} k+\frac{5}{12} k^{2}-\frac{11}{36} k^{3}\right) n^{3}+\ldots$

Consider now the number $D(4, n, K)$ of distributions with a dummy player with $n_{1} \leq K$. All we have to do is to add to the above set of inequalities $n_{2}+n_{3}>n / 2$. Replacing $K$ by $k n$ in the quasi polynomials associated with this new set on inequalities gives:

For $\frac{1}{4} \leq k<\frac{1}{3}: D(4, n, k)=\left(-\frac{1}{192}+\frac{1}{16} k-\frac{1}{4} k^{2}+\frac{1}{3} k^{3}\right) n^{3}+\ldots$

For $\frac{1}{3} \leq k \leq \frac{1}{2}: D(4, n, k)=\left(\frac{13}{576}-\frac{3}{16} k+\frac{1}{2} k^{2}-\frac{5}{12} k^{3}\right) n^{3}+\ldots$

We finally obtain:

For $\frac{1}{4} \leq k<\frac{1}{3}$ :

$$
P(4, \infty, k)=\frac{D(4, \infty, k)}{T(4, \infty, k)}=\frac{-\frac{1}{192}+\frac{1}{16} k-\frac{1}{4} k^{2}+\frac{1}{3} k^{3}}{-\frac{1}{144}+\frac{1}{12} k-\frac{1}{3} k^{2}+\frac{4}{9} k^{3}}=\frac{\frac{(4 k-1)^{3}}{192}}{\frac{(4 k-1)^{3}}{144}}=\frac{3}{4},
$$

and for $\frac{1}{4} \leq k<\frac{1}{3}$ :

$$
P(4, \infty, k)=\frac{D(4, \infty, k)}{T(4, \infty, k)}=\frac{\frac{13}{576}-\frac{3}{16} k+\frac{1}{2} k^{2}-\frac{5}{12} k^{3}}{\frac{1}{48}-\frac{1}{6} k+\frac{5}{12} k^{2}-\frac{11}{36} k^{3}}=\frac{240 k^{3}-288 k^{2}+108 k-13}{4\left(44 k^{3}-60 k^{2}+24 k-3\right)} .
$$

Proposition 7 In a 5-player WMG with n large, the probability of having at least one dummy player depends on $k$ as shown in the following representation:

$$
\begin{gathered}
P(5, \infty, k)=0 \text { for } \frac{1}{5}<k<\frac{1}{4} \\
=\frac{5(4 k-1)^{3}(44 k-23)}{32\left(655 k^{4}-780 k^{3}+330 k^{2}-60 k+43\right)} \text { for } \frac{1}{4}<k<\frac{1}{3} \\
=\frac{-5\left(3264 k^{4}-3840 k^{3}+1440 k^{2}-192 k+5\right)}{96\left(155 k^{4}-300 k^{3}+210 k^{2}-60 k+6\right)} \text { for } \frac{1}{3}<k<\frac{1}{2} .
\end{gathered}
$$

Proposition 8 In a 6-player WMG with n large, the probability of having at least one dummy player depends on $k$ as shown in the following representation:

$$
\begin{aligned}
& P(6, \infty, k)=\frac{5}{12} \text { for } \frac{1}{6}<k<\frac{1}{5} \\
& =\frac{186120 k^{5}-192600 k^{4}+79200 k^{3}-16200 k^{2}+1650 k-67}{12\left(10974 k^{5}-12270 k^{4}+5340 k^{3}-1140 k^{2}+120 k-5\right)} \text { for } \frac{1}{5}<k<\frac{1}{4} \\
& =-\frac{5034240 k^{5}-7027200 k^{4}+3916800 k^{3}-1094400 k^{2}+153600 k-8669}{768\left(2193 k^{5}-3465 k^{4}+2130 k^{3}-630 k^{2}+90 k-5\right)} \text { for } \frac{1}{4}<k<\frac{3}{10} \\
& =\frac{5\left(1153152 k^{5}-1834560 k^{4}+1160640 k^{3}-364320 k^{2}+56760 k-3515\right)}{768\left(2193 k^{5}-3465 k^{4}+2130 k^{3}-630 k^{2}+90 k-5\right)} \text { for } \frac{3}{10}<k<\frac{1}{3} \\
& =-\frac{5\left(282240 k^{5}-486720 k^{4}+331200 k^{3}-110880 k^{2}+18360 k-1211\right)}{768\left(237 k^{5}-585 k^{4}+570 k^{3}-270 k^{2}+60 k-5\right)} \text { for } \frac{1}{3}<k<\frac{3}{8} \\
& =\frac{5\left(307584 k^{5}-619200 k^{4}+498240 k^{3}-200160 k^{2}+39960 k-3163\right)}{768\left(237 k^{5}-585 k^{4}+570 k^{3}-270 k^{2}+60 k-5\right)} \text { for } \frac{3}{8}<k<\frac{1}{2} \text {. }
\end{aligned}
$$


Table 5

Exact probability $P(m, \infty, k)$ of having a dummy player as a function of $k$ for large $n$ and $m=4,5,6$.

\begin{tabular}{||c||c|c|c||}
\hline$k$ & 4-player WMG & 5-player WMG & 6-player WMG \\
\hline 0.23 & - & 0 & 0.381 \\
0.25 & 0.750 & 0 & 0.282 \\
0.27 & 0.750 & 0.060 & 0.269 \\
0.29 & 0.750 & 0.174 & 0.311 \\
0.31 & 0.750 & 0.270 & 0.354 \\
0.33 & 0.750 & 0.344 & 0.391 \\
0.35 & 0.748 & 0.403 & 0.418 \\
0.37 & 0.737 & 0.452 & 0.439 \\
0.39 & 0.718 & 0.492 & 0.455 \\
0.41 & 0.693 & 0.525 & 0.469 \\
0.43 & 0.662 & 0.549 & 0.482 \\
0.45 & 0.624 & 0.563 & 0.495 \\
0.47 & 0.580 & 0.563 & 0.505 \\
0.49 & 0.529 & 0.547 & 0.505 \\
0.50 & 0.500 & 0.530 & 0.497 \\
\hline
\end{tabular}

Table 6

Simulated ${ }^{7}$ probability $P(m, 9999, k)$ of having a dummy player as a function of $k$ and $m$.

\begin{tabular}{||c||c|c|c|c||}
\hline$k$ & 7-player WMG & 8-player WMG & 9-player WMG & 10-player WMG \\
\hline 0.23 & 0.199 & 0.175 & 0.146 & 0.099 \\
0.25 & 0.235 & 0.198 & 0.163 & 0.117 \\
0.27 & 0.259 & 0.221 & 0.182 & 0.125 \\
0.29 & 0.290 & 0.238 & 0.192 & 0.133 \\
0.31 & 0.319 & 0.255 & 0.205 & 0.143 \\
0.33 & 0.346 & 0.271 & 0.211 & 0.152 \\
0.35 & 0.365 & 0.283 & 0.219 & 0.157 \\
0.37 & 0.376 & 0.297 & 0.223 & 0.160 \\
0.39 & 0.387 & 0.302 & 0.228 & 0.163 \\
0.41 & 0.393 & 0.313 & 0.234 & 0.165 \\
0.43 & 0.402 & 0.322 & 0.238 & 0.167 \\
0.45 & 0.413 & 0.333 & 0.243 & 0.174 \\
0.47 & 0.422 & 0.340 & 0.247 & 0.177 \\
0.49 & 0.428 & 0.347 & 0.252 & 0.180 \\
0.50 & 0.435 & 0.435 & 0.258 & 0.1775 \\
\hline
\end{tabular}

${ }^{7} 10000$ simulations for $m<11$ and 50000 when $m \geq 11$. 


\section{Concluding remark}

We have shown in this paper that the probability of having at least one dummy player in Weighted Majority Games with a small number of player is very high. This probability can reach about $50 \%$ for 4,5 or 6 players ; for more than 6 players, the probability decreases but we have to consider more than 15 players for obtaining results lower than $1 \%$. Of course, it can be suspected that our probabilistic assumption (all admissible weight distributions are supposed to be equally likely to occur) could tend to exaggerate the probability of having a dummy. We have proved however that, for a very small number of players, the introduction of some degree of homogeneity in the distribution of the weights has a weak impact on this probability.

Finally, it is worth to emphasize that our results are limited to majority games, in which the quota for a proposition to be approved is equal to $50 \%$ of the total weight. It should be of interest to consider the impact of the quota value on the probability of having a dummy player. We plan to study this question in another paper.

\section{Appendix: Proof of Proposition 2}

(i) In order to characterize the distributions of the $n_{i}$ 's for which $J 5$ is a dummy player, we consider the set of coalitions to which $J 5$ is susceptible to belong: $\{J 1, J 5\},\{J 2, J 5\},\{J 3, J 5\}$, $\{J 4, J 5\},\{J 1, J 2, J 5\},\{J 1, J 3, J 5\},\{J 1, J 4, J 5\},\{J 2, J 3, J 5\},\{J 2, J 4, J 5\},\{J 3, J 4, J 5\}$, $\{J 1, J 2, J 3, J 5\},\{J 1, J 2, J 4, J 5\},\{J 1, J 3, J 4, J 5\},\{J 2, J 3, J 4, J 5\}$ and $\{J 1, J 2, J 3, J 4, J 5\}$. Consider first the two-player coalitions; $J 5$ is a dummy player if these coalitions are loosing (if not, a zero power for $J 5$ would imply that a coalition with only one player is winning, contradicting our assumptions) and these two-player coalitions will be loosing if $n_{1}+n_{5}<Q$. Consider now the coalitions $\{J 1, J 2, J 5\}$ and $\{J 1, J 3, J 5\}$; these coalitions are necessarily winning (recall that $n_{1} \geq n_{2} \geq n_{3} \geq n_{4} \geq n_{5} \geq 1$, by assumption (2)). J5 is a dummy if we have $n_{1}+n_{3} \geq Q$ (which implies $n_{1}+n_{2} \geq Q$ ). The next three-player coalitions $\{J 1, J 4, J 5\}$, $\{J 2, J 3, J 5\},\{J 2, J 4, J 5\}$ and $\{J 3, J 4, J 5\}$ can be winning or loosing. Hence, $J 5$ is a dummy if: $\left(n_{1}+n_{4}+n_{5}<Q\right.$ or $\left.n_{1}+n_{4} \geq Q\right)$ and $\left(n_{2}+n_{3}+n_{5}<Q\right.$ or $\left.n_{2}+n_{3} \geq Q\right)$ and $\left(n_{2}+n_{4}+n_{5}<Q\right.$ or $\left.n_{2}+n_{4} \geq Q\right)$ and $\left(n_{3}+n_{4}+n_{5}<Q\right.$ or $\left.n_{3}+n_{4} \geq Q\right)$. Finally, consider coalitions with four or five players, which are winning coalitions. $J 5$ is a dummy player is $n_{2}+n_{3}+n_{4} \geq Q$. To summing up, $J 5$ is a dummy player if and only if we have:

$n_{1}+n_{5}<Q$ and $n_{1}+n_{3} \geq Q$ and $\left(n_{1}+n_{4}+n_{5}<Q\right.$ or $\left.n_{1}+n_{4} \geq Q\right)$ and $\left(n_{2}+n_{3}+n_{5}<Q\right.$ or $\left.n_{2}+n_{3} \geq Q\right)$ and $\left(n_{2}+n_{4}+n_{5}<Q\right.$ or $\left.n_{2}+n_{4} \geq Q\right)$ and $\left(n_{3}+n_{4}+n_{5}<Q\right.$ or $\left.n_{3}+n_{4} \geq Q\right)$ and $n_{2}+n_{3}+n_{4} \geq Q$.

Since $\sum_{i} n_{i}=n$, these inequalities can be written in the following way:

$n_{1}+n_{5}<Q$ and $n_{1}+n_{3} \geq Q$ and $\left(n_{2}+n_{3} \geq Q^{*}\right.$ or $\left.n_{1}+n_{4} \geq Q\right)$ and $\left(n_{1}+n_{4} \geq Q^{*}\right.$ or

$\left.n_{2}+n_{3} \geq Q\right)$ and $\left(n_{1}+n_{3} \geq Q^{*}\right.$ or $\left.n_{2}+n_{4} \geq Q\right)$ and $\left(n_{1}+n_{2} \geq Q^{*}\right.$ or $\left.n_{3}+n_{4} \geq Q\right)$ and $n_{1}+n_{5}<Q^{*}$

Eliminating redundant inequalities, we obtain:

$n_{1}+n_{5}<Q^{*}$ and $n_{1}+n_{3} \geq Q$ and $\left(n_{2}+n_{3} \geq Q\right.$ or $\left.n_{1}+n_{4} \geq Q\right)$ and $n_{1}+n_{3} \geq Q$ and 
$n_{1}+n_{2} \geq Q$,

which can be reduced to:

$\left(n_{1}+n_{5}<Q^{*}\right.$ and $\left.n_{2}+n_{3} \geq Q\right)$ or $\left(n_{1}+n_{5}<Q^{*}\right.$ and $\left.n_{1}+n_{4} \geq Q\right)$.

As $n_{2}+n_{3} \geq Q$ implies $n_{1}+n_{5}<Q^{*}$ and $n_{1}+n_{5}<Q^{*}$ is equivalent to $n_{2}+n_{3}+n_{4} \geq Q$, we finally obtain:

$n_{2}+n_{3} \geq Q$ or $\left(n_{2}+n_{3}+n_{4} \geq Q\right.$ and $\left.n_{1}+n_{4} \geq Q\right)$, in accordance with Proposition 2 (i). Furthermore, it follows from Proposition 1 that $J 4$ and $J 5$ are both dummy players if and only if $n_{2}+n_{3} \geq Q$.

(ii) Proceeding as above, it is easily checked that $J 6$ is a dummy player if and only if a) all the two-player coalitions including $J 6$ are loosing, b) either $\{J 1, J 2, J 6\},\{J 1, J 3, J 6\},\{J 1, J 4, J 6\}$, $\{J 1, J 5, J 6\},\{J 2, J 3, J 6\}$ are loosing or (respectively) $\{J 1, J 2\},\{J 1, J 3\},\{J 1, J 4\},\{J 1, J 5\}$, $\{J 2, J 3\}$ are winning, c) either $\{J 1, J 4, J 5, J 6\},\{J 2, J 3, J 4, J 6\},\{J 2, J 3, J 5, J 6\},\{J 2, J 4, J 5, J 6\}$, $\{J 3, J 4, J 5, J 6\}$ are loosing or (respectively) $\{J 1, J 4, J 5\},\{J 2, J 3, J 4\},\{J 2, J 3, J 5\},\{J 2, J 4, J 5\}$, $\{J 3, J 4, J 5\}$ are winning, d) $\{J 1, J 2, J 3\},\{J 1, J 2, J 4\},\{J 1, J 2, J 5\},\{J 1, J 3, J 4\},\{J 1, J 3, J 5\}$ are winning, e) $\{J 1, J 2, J 3, J 4\},\{J 1, J 2, J 3, J 5\},\{J 1, J 2, J 4, J 5\},\{J 1, J 3, J 4, J 5\},\{J 2, J 3, J 4, J 5\}$ are winning and f) $\{J 1, J 2, J 3, J 4, J 5\}$ is winning. This implies a) $\left.n_{1}+n_{6}<Q, \mathrm{~b}\right)\left(n_{1}+n_{2}+n_{6}<\right.$ $Q$ or $\left.n_{1}+n_{2} \geq Q\right)$ and $\left(n_{1}+n_{3}+n_{6}<Q\right.$ or $\left.n_{1}+n_{3} \geq Q\right)$ and $\left(n_{1}+n_{4}+n_{6}<Q\right.$ or $\left.n_{1}+n_{4} \geq Q\right)$ and $\left(n_{1}+n_{5}+n_{6}<Q\right.$ or $\left.n_{1}+n_{5} \geq Q\right)$ and $\left(n_{2}+n_{3}+n_{6}<Q\right.$ or $\left.n_{2}+n_{3} \geq Q\right)$, c) $\left(n_{1}+n_{4}+n_{5}+n_{6}<Q\right.$ or $\left.n_{1}+n_{4}+n_{5} \geq Q\right)$ and $\left(n_{2}+n_{3}+n_{4}+n_{6}<Q\right.$ or $\left.n_{2}+n_{3}+n_{4} \geq Q\right)$ and $\left(n_{2}+n_{3}+n_{5}+n_{6}<Q\right.$ or $\left.n_{2}+n_{3}+n_{5} \geq Q\right)$ and $\left(n_{2}+n_{4}+n_{5}+n_{6}<Q\right.$ or $\left.n_{2}+n_{4}+n_{5} \geq Q\right)$ and $\left(n_{3}+n_{4}+n_{5}+n_{6}<Q\right.$ or $n_{3}+n_{4}+n_{5} \geq Q$ ), d) $n_{1}+n_{3}+n_{5} \geq Q$, e) $n_{2}+n_{3}+n_{4}+n_{5} \geq Q$ and f) $n_{1}+n_{2}+n_{3}+n_{4}+n_{5} \geq Q$. The reduction of this set of inequalities leads to the six cases given in Proposition 2 (ii). To complete the proof, it remains to observe that, in case $4, J 4, J 5$ and $J 6$ are dummy players (by Proposition 1); and if $J 6$ is a dummy and $J 4$ is not $\left(n_{2}+n_{3}<Q\right)$, then it results from part (i) of Proposition 2 that $J 5$ is also a dummy player if and only if $n_{1}+n_{4} \geq Q$ and $n_{2}+n_{3}+n_{4} \geq Q$. $\square$ 